\title{
Evaluación y abordaje de la depresión y la ansiedad en triaje de urgencias: implementación de la GI-mhGAP 2.0
}

\section{Evaluation and approach of depression and anxiety in emergency triage: implementation of the GI-mhGAP 2.0}

\author{
Alma H. Guerrero-Martínez ${ }^{1 *}$, Brenda A. Barrera-Flores ${ }^{1}$, Ixchel Calixto-Molina ${ }^{1}$, Lesly P. Díaz-Vásquez ${ }^{1}$, \\ Viridiana G. Eslava-Torres ${ }^{1}$, Guadalupe X. Ortega-Ramírez', Katya F. Cabañas-Moreno', \\ César De Lucio-Ramírez ${ }^{1}$, Wendy V. Bernal-Ruíz ${ }^{1}$ y Edgar Landa-Ramírez ${ }^{1,2}$ \\ ${ }^{1}$ División de Urgencias y Observación, Programa de Psicología Urgencias, Hospital General Dr. Manuel Gea González; ${ }^{2}$ Programa de Psicología, \\ Facultad de Psicología, Universidad Nacional Autónoma de México. Ciudad de México, México
}

\section{Introducción}

Más del $25 \%$ de las visitas al departamento de emergencias son realizadas por pacientes con problemas de salud mental comórbidos, como la ansiedad y la depresión ${ }^{1}$. No obstante, en el departamento de emergencias no se realizan pruebas de detección de problemas de salud mental a menos que sea la queja principal, debido a múltiples factores, como prioridades de atención o recursos limitados ${ }^{2}$. Esto puede provocar la saturación del servicio, numerosas evaluaciones a altos costos, mayor número de reingresos y más tiempo de hospitalización ${ }^{3-7}$, ya que las personas con sintomatología ansiosa o depresiva pueden utilizar el departamento de emergencias para preocupaciones que no son urgentes $^{8}$. En este sentido, la Guía de Intervención del Programa de Acción Global de Salud Mental (GI-mhGAP) resulta una herramienta de evaluación e intervención útil para estas afecciones, principalmente en los países con bajos y medianos ingresos como es México ${ }^{9}$. Por lo tanto, evaluar con la Gl-mhGAP, adaptándola a este contexto, permitirá identificar necesidades y barreras para la ampliación y la mejora del departamento de emergencias ${ }^{10}$.
El objetivo de este estudio fue explorar la implementación y la adaptación de la Gl-mhGAP versión 2.0 para identificar sintomatología depresiva y ansiosa en pacientes que llegan al triaje de urgencias médicas en un hospital en México.

\section{Método}

Estudio con enfoque cuantitativo y alcance exploratorio, no experimental, analítico-observacional. La muestra se conformó por 200 pacientes del triaje de urgencias médicas del Hospital General Dr. Manuel Gea González, en la Ciudad de México. Para evaluar la sintomatología ansiosa y depresiva se realizaron dos algoritmos de acuerdo con los criterios de la Gl-mhGAP 2.0. Se evaluaron datos sociodemográficos, condición médica, evaluación fisiológica y percepción del médico para identificar estas sintomatologías. Asimismo, se aplicó la Escala Hospitalaria de Ansiedad y Depresión (HADS) y se elaboró el algoritmo del semáforo para abordar a los pacientes: en verde se brindaron consejos de salud mental generales, en amarillo se brindaron recomendaciones breves y se fomentaron redes de apoyo, y en rojo se realizó una intervención

\section{Correspondencia:}

*Alma H. Guerrero-Martínez

E-mail: gueerreromartinez.almahaide1@gmail.com bajo la licencia CC BY-NC-ND (http://creativecommons.org/licenses/by-nc-nd/4.0/).
Fecha de recepción: 30-11-2021

Fecha de aceptación: 15-12-2021 DOI: 10.24875/REIE.21000209
Disponible en internet: 07-03-2022

Rev Educ Investig Emer. 2022;4(Supl 1):29-31 www.medicinadeemergencias.com México SA de CV. Este es un artículo open access 


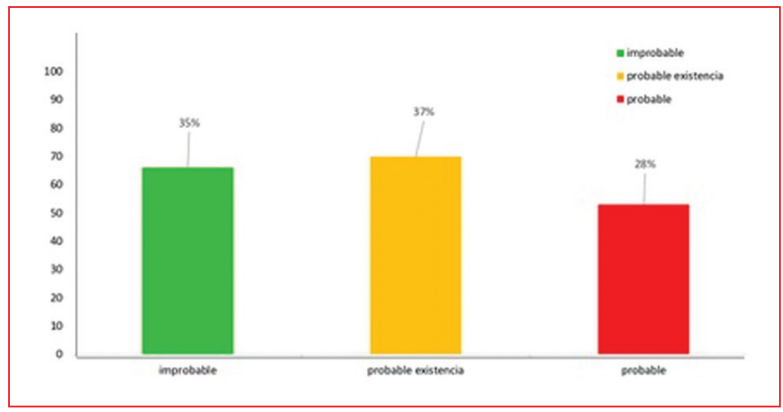

Figura 1. Presencia de ansiedad de acuerdo con el semáforo de manejo de los participantes del Hospital General "Dr. Manuel Gea Gonzales" de la Ciudad de México (n=189).

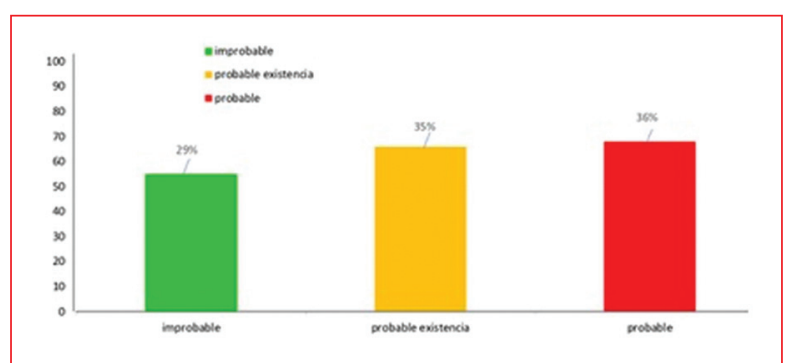

Figura 2. Presencia de depresión de acuerdo al semáforo de manejo de los participantes del Hospital General "Dr. Manuel Gea Gonzales" de la Ciudad de México (n=189).

Tabla 1. Concordancia entre médico y psicólogo

\begin{tabular}{|l|c|c|c|c|c|c|}
\hline \multirow{2}{*}{ Variable } & \multicolumn{6}{|c|}{ Identificación del médico/psicólogo } \\
\cline { 2 - 8 } & $\begin{array}{c}\text { Sí/ } \\
\text { Sí }\end{array}$ & $\begin{array}{c}\text { No/ } \\
\text { No }\end{array}$ & $\begin{array}{c}\text { Sí/ } \\
\text { No }\end{array}$ & $\begin{array}{c}\text { No/ } \\
\text { Sí }\end{array}$ & Kappa & IC 95\% \\
\hline Depresión & 12 & 108 & 17 & 36 & 0.130 & $(-0.024 ; 0.28)$ \\
\hline Ansiedad & 29 & 84 & 27 & 33 & 0.229 & $(-0.8 ; 0.38)$ \\
\hline
\end{tabular}

IC: intervalo de confianza $95 \%$

breve, psicoeducación o canalización a instituciones públicas de atención especializada (Figs. 1 y 2).

Se realizaron análisis de tendencia central y dispersión de los datos, así como coeficiente kappa de Cohen para las variables.

\section{Resultados}

Del total de las evaluaciones realizadas, 189 tenían información completa; el $35.9 \%$ cumplieron con criterios del algoritmo para aplicar la HADS de depresión y el 39\% para ansiedad. En el caso de la depresión, el $27.5 \%$ presentaron puntajes por encima del punto de corte, indicando depresión, y el $8.4 \%$ obtuvieron puntajes inferiores al punto de corte. El $35.8 \%$ presentaron un puntaje que indicó presencia de ansiedad, y el $3.2 \%$ no lo presentaron.

Para la percepción de sintomatología depresiva se encontró un punto de acuerdo entre médico y psicólogo de 12 veces, y de desacuerdo de 108 veces, obteniendo así un acuerdo mínimo entre el juicio de estos profesionales de $\mathrm{K}=0.130$, con un intervalo de confianza del $95 \%$ (IC95\%) de -0.024 a 0.28 . Para la sintomatología ansiosa se encontró 29 veces de concordancia y 84 veces de inconsistencia, obteniendo un acuerdo justo, con $\mathrm{k}=0.229$ y un IC95\% de 0.08 a 0.38 (Tabla 1).

De acuerdo con la pertinencia del caso, para ambas sintomatologías, se brindaron consejos de salud mental en general (semáforo verde), recomendaciones breves y fomentar las redes de apoyo (semáforo amarillo), o intervención breve y posibilidad de interconsulta y recomendaciones breves (semáforo rojo).

\section{Conclusiones}

Con la implementación de la adaptación de la Gl-mhGAP 2.0 fue posible identificar y brindar manejo de la sintomatología depresiva y ansiosa a los pacientes que acudieron al triaje de urgencias médicas, así como realizar inferencias iniciales para desarrollar líneas de investigación y tratamiento en la población de triaje. Sin embargo, a pesar de las virtudes, el tamaño de la muestra en comparación con otros estudios anteriores es relativamente pequeño, y por lo tanto no se puede generalizar al resto de la población. Se sugiere una muestra mayor tomando en cuenta el total de las personas que visitan el triaje de urgencias médicas durante las 24 horas de servicio, además de considerar días de la semana y épocas del año. Asimismo, se debe capacitar al personal sobre esta herramienta y realizar una validación de la adaptación de la Gl-mhGAP 2.0.

\section{Agradecimientos}

Agradecemos al equipo del departamento de urgencias médicas y de psicología de urgencias del Hospital General Dr. Manuel Gea González, por el apoyo y por brindar las condiciones necesarias para que este trabajo fuera posible. 


\section{Financiamiento}

Este proyecto no tiene relación económica y se encuentra fuera de cualquier tipo de patrocinio o financiamiento.

\section{Conflicto de intereses}

Los autores declaran no tener ningún conflicto de intereses.

\section{Responsabilidades éticas}

Protección de personas y animales. Los autores declaran que para esta investigación no se han realizado experimentos en seres humanos ni en animales.

Confidencialidad de los datos. Los autores declaran que han seguido los protocolos de su centro de trabajo sobre la publicación de datos de pacientes.

Derecho a la privacidad y consentimiento informado. Los autores han obtenido el consentimiento informado de los pacientes y/o sujetos referidos en el artículo. Este documento obra en poder del autor de correspondencia.

\section{Bibliografía}

1. Capp R, Hardy R, Lindrooth R, Wiler J. National trends in emergency department visits by adults with mental health disorders. J Emerg Med. 2016;51:131-5.e1.

2. Horowitz LM, Bridge JA, Teach SJ, Ballard E, Klima J, Rosenstein DL, et al. Ask Suicide-Screening Questions (ASQ): a brief instrument for the pediatric emergency department. Arch Pediatr Adolesc Med. 2012;166:1170-6.

3. Ballou S, Mitsuhashi S, Sankin LS, Petersen TS, Zubiago J, Lembo C, et al. Emergency department visits for depression in the United States from 2006 to 2014. Gen Hosp Psychiatry. 2019;59:14-9.

4. Edmondson D, Newman JD, Chang MJ, Wyer P, Davidson KW. Depression is associated with longer emergency department length of stay in acute coronary syndrome patients. BMC Emerg Med. 2012;12:14.

5. Meltzer AC, Bregman B, Blanchard J. Depression is associated with repeat emergency department visits in patients with non-specific abdominal pain. West J Emerg Med. 2014;15:325-8.

6. Musey PI Jr, Lee JA, Hall CA, Kline JA. Anxiety about anxiety: a survey of emergency department provider beliefs and practices regarding anxiety-associated low risk chest pain. BMC Emerg Med. 2018;18:10.

7. Urgencias. Gob.mx. (Consultado el 26-11-2021.) Disponible en: http:// data.salud.cdmx.gob.mx/portal/me

8. Behr JG, Diaz R. Emergency department frequent utilization for non-emergent presentments: results from a regional urban trauma center study. PLoS One. 2016;11:e0147116.

9. World Health Organization. MhGAP: Mental Health Gap Action Programme: scaling up care for mental, neurological and substance use disorders. Apps.who.int, 2008. (Consultado el 26-11-2021.) Disponible en: apps. who.int/iris/handle/10665/43809.

10. World Health Organization. MhGAP Intervention Guide Mental Health Gap Action Programme Version 2.0 for mental, neurological and substance use disorders in non-specialized health settings. Disponible en: https:// iris.paho.org/handle/10665.2/34071 